\title{
Case report: A rare case of vaginal leiomyoma
}

\author{
Rakhee R. Sahu \\ Consultant, Dept. of Obstetrics and Gynaecology, Dr. L. H. Hiranandani Hospital, Powai, Mumbai, Maharashtra, India \\ *Corresponding Author: \\ Email: rakhee.sahu@hiranandanihospital.org

\begin{abstract}
Leiomyoma is the most common benign tumor of uterus and rarely it may be seen in other parts female reproductive system like the round ligament, utero-sacral ligament, inguinal canal and vagina. There are only few cases reported in literature about vaginal origin fibroid ${ }^{1}$. These vaginal tumors arise most commonly from the anterior vaginal wall causing varied clinical presentations. We report a case of vaginal lateral wall fibroid of $8 \mathrm{~cm}$ size and extending into ischiorectal space. The vaginal mass was surgically excised and histopathology confirmed the diagnosis of leiomyoma.
\end{abstract}

Keywords: Vaginal leiomyoma, Perineal mass.

\section{Introduction}

Leiomyomas are the commonly presenting tumors of female genital tract but occurrence in vagina is very rare. Vaginal leiomyomas usually arise from anterior vaginal wall as a single solid mass and occurrence in the lateral vaginal wall is rare. Vaginal fibroids have varied clinical presentations depending on its location, size and vascularity.

\section{Case Report}

We report a rare case of a vaginal leiomyoma in a young woman of 33 years of age who came with complain of swelling in the right perineal region which was gradually increasing in size over 3 years. She complained of difficulty in sitting and dysparunia since 3 months. She had 3 full-term normal deliveries and last delivery was 6 years ago. She had no menstrual complains and no past history of any significant medical or surgical illness. On examination, her vital parameters were normal. On local examination, a lump measuring $8 \times 8 \mathrm{~cm}$ was seen in the right perineum extending from the right labia majora till the ischial spine. On per speculum examination, a solitary mass was seen in lower half of vagina, arising from right lateral vaginal wall and extending laterally. The upper pole of the solid, firm globular mass was palpable and the cervix was seen separately from the vaginal mass. On per vaginal examination, uterus was corresponding to 6 weeks and no adnexal mass was palpable. On per rectal examination, the rectal mucosa was free and the solid mass was palpable in the right paravaginal space. Provisional diagnosis of right paravaginal mass, ischiorectal mass of unknown origin or chronic bartholin gland cyst was made.

Her blood investigations - CBC, sugar, S. creatinine and pap smear reports were normal. MRI pelvis reported a 7.8 x 5.9 × $4.5 \mathrm{~cm}$ well defined exophytic soft tissue arising from right side of vagina and extending laterally into ischiorectal space. The mass showed heterogeneous low signal intensity on the T2- weighted images. The fat planes with rectum and adjacent perineal structures were maintained, suggestive of benign lesion. There was $5.3 \times 3.5 \mathrm{~cm}$ hypodense lesion arising from right lateral wall of uterus suggestive of subserosal fibroid and both ovaries /adnexa were normal. Surgical excision of the perineal mass was done through vagina. Incision was taken at the muco-cutaneous junction on right lateral wall of vagina. The mass was enucleated from the ischiorectal space which was firm in consistency and well circumcised. The perineal muscle was approximated with 2-0 vicryl interrupted sutures. Vaginal mucosa was closed with 2-0 vicryl interrupted sutures. The cut section of the mass showed whorled appearance and histopathology confirmed it as benign leiomyoma.

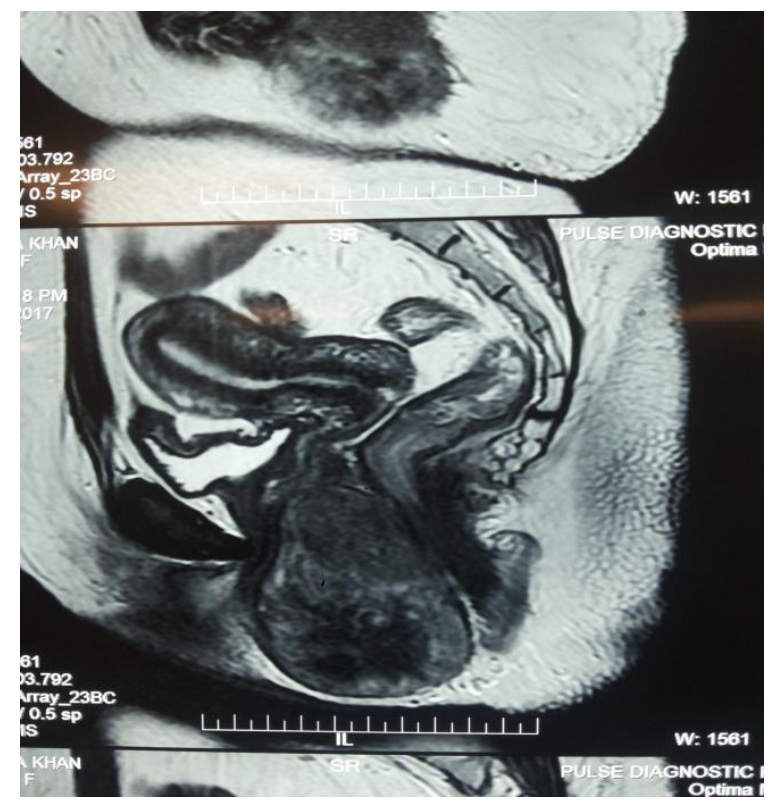

Fig. 1: MRI image of vaginal leiomyoma

MRI image showing the mass arising from the vaginal wall 


\section{Discussion}

Leiomyoma is the most common tumor of the uterus and the female pelvis but occurrence in the vagina is rare. Fibroid or leiomyoma, a benign overgrowth of mainly of the smooth muscle cells but containing varying amounts of fibrous connective tissue.

Leiomyomas are almost always in the myometrium, but it may develop in any part of the genital tract like cervix, broad ligament, round ligament, inguinal ligament and rarely vagina. About only 300 case repots of vaginal fibroid have been published since the first detected case back in 1733 by Denys de Leyden. ${ }^{1}$

We report a rare case of vaginal fibroid arising from lower $1 / 3^{\text {rd }}$ of lateral vaginal wall mucosa presenting as a large perineal mass. She also had a subserosal uterine fibroid for which she was asymptomatic.

Vaginal leiomyomas are commonly seen in the age group ranging from 35 to 50 years and are reported to be more common among caucasian women. ${ }^{2}$ Vaginal fibroids are usually present in the anterior vaginal wall but can arise from lateral or posterior vaginal wall. ${ }^{2}$

The anterior vaginal fibroids can clinically present with varied symptoms like difficulty in urination, frequency of urination due to uretheral compression, dyspareunia, vaginal pain, pelvic pain or vaginal bleeding. They are often misdiagnosed as cystocele, paraurethral cyst, urethral diverticulum, Gartner's duct cyst, bartholin's cystvaginal cyst, cervical fibroid, epidermal inclusion cyst or pararectal mass. Surgical excision through vaginal route is preferred. If it is a large vaginal mass extending into the pelvis, abdominal route may be required.

During surgical removal of anterior vaginal wall fibroid, there is increased risk of trauma to the urethra and urinary bladder base ${ }^{3}$. So, adequate precautions like Foley's catheterization and assistance from Urologist should be taken to prevent damage to the urethra and base of urinary bladder.

These vaginal fibroids are usually single, wellcircumscribed, solid or cystic, slow growing and benign but sarcomatous changes have been reported. ${ }^{4}$

Transperineal and abdominal ultrasound and MRI can be useful to estimate the extent and involvement of the mass. In magnetic resonance imaging (MRI), fibroids appear as solid masses of low intensity in T1 and T2 weighted images. Leiomyosarcomas in MRI would show characteristic high T2 signal intensity with heterogeneous areas of hemorrhage and necrosis. ${ }^{5}$

We report a case of vaginal fibroid arising from the lateral wall of vagina extending into ischiorectal space, which is a rare entity.

Conflict of Interest: The authors declare they have no conflict of interest.

\section{References}

1. Young SB, Rose PG, Reuter KL. Vaginal fibromyomata: Two cases with preoperative assessment, resection and reconstruction. Obstet Gynecol. 1991;78:972-4.

2. Elsayes KM, Narra VR, Dillman JR, Velcheti V, Hameed $\mathrm{O}$, Tongdee R, et al. Vaginal Masses: Magnetic Resonance Imaging Features with Pathologic Correlation. Acta Radiol. 2007;8:921-33.

3. Blaivas J.G., Flisser A.J., Bleustein C.B., Panagopoulos G. Periurethral masses: etiology and diagnosis in a large series of women. Obstet Gynecol. 2004;103(5 Pt 1):842847.

4. Cobanoğlu O, Gürkan Zorlu C, Ergun Y, Kutluay L. Leiomyosarcoma of the vagina. Eur J Obstet Gynecol Reprod Biol. 1996;70:205-7.

5. Shadbolt CL, Coakley FV, Qayyum A, Donat SM. MRI of vaginal leiomyomas. J Comput Assist Tomogr. 2001;25:355-7. 